\title{
SPECTROSCOPY OF FEW-ELECTRON QUANTUM DOTS
}

\author{
D. Heitmann ${ }^{a}$, V. Gudmundsson ${ }^{b}$, L. Rolf $^{a}$, C. SchülleR ${ }^{a}$, \\ C. STEINEBACH ${ }^{a}$ AND E. UlRIChS ${ }^{a}$ \\ ${ }^{a}$ Institut für Angewandte Physik und Zentrum für Mikrostrukturforschung \\ Universität Hamburg, Jungiusstraße 11, 20355 Hamburg, Germany \\ ${ }^{b}$ Science Institute, University of Iceland, Dunhaga 3, 107 Reykjavik, Iceland
}

Advances in submicron technology make it possible to realize man-made low-dimensional electronic systems with quantum confined energy states. Superimposing lateral potential onto two-dimensional electron systems for example in AlGaAs-GaAs systems allows one to prepare quantum wires and dots. We will discuss modulation-doped systems. Then, with typical confinement energies in the $\mathrm{meV}$ regime, far-infrared and resonant Raman spectroscopy give direct access to the quantum confined energy levels of the systems. One is now approaching the limit to prepare quantum dots with well defined small numbers of electrons per dot, $N=1,2,3 \ldots$ It becomes thus possible to perform a kind of "atomic" spectroscopy in these systems.

PACS numbers: $72.15 . \mathrm{Rn}, 73.20 . \mathrm{Dx}, \cdot 73.20 . \mathrm{Mf}, 78.30 . \mathrm{Fs}$

\section{Introduction}

The progress and success of physics in layered two-dimensional (2D) semiconductor structures with quantum confined energy states has challenged many scientists to prepare and study systems with further reduced dimensionality, specifically quantum wires, quantum dots, and antidots $[1,2]$. In these systems, due to an ultrafine lateral confinement, the originally free dispersions of the electrons in the lateral directions are also quantized. An ultimate limit is a quantum dot, where, induced by a confining potential in both the $x$ and $y$ directions, artificial "atoms" with a totally discrete energy spectrum are formed [3-9]. The growth direction is labeled $z$ in the following. We will discuss modulation-doped systems. Then information on the quantum confined energy levels in these low-dimensional systems can be obtained from far-infrared (FIR) and resonant Raman spectroscopy. It turns out that the dynamic response of these systems exhibits a very interesting complex interplay of atom-like single-particle and many-body effects. Spectroscopy on low-dimensional systems is now a very wide field. We would like to review here some selected older and newer experiments to give an introduction into this field. The review and the list of references is by no means complete. It rather should serve as a starting point for further reading. 


\section{Preparation}

Two examples of quantum dot structures, which were prepared starting from modulation-doped AlGaAs-GaAs heterostructures, are sketched in Fig. 1. For the "deep-mesa-etched" quantum dots in Fig. 1a an array of photoresist dots (with typical periods of $a=200$ to $1000 \mathrm{~nm}$ both in the $x$ and $y$ direction) is prepared by a holographic double exposure [4]. Using an anisotropic plasma etching process, deep grooves are etched all the way through the GaAs cap layer, the Si-doped $\mathrm{AlGaAs}$ layer, and the undoped AlGaAs spacer layer into the active GaAs, leaving dots with geometrical dimensions of about 100 to $500 \mathrm{~nm}$. The actual "electronic" width of the electron systems is smaller than the geometrical width, arising from a lateral edge depletion of 100 to $200 \mathrm{~nm}$. Actually, one can prepare samples which have, in the dark, no mobile electrons. Via the persistent photoeffect one can then increase the number of electrons per dot $N$ in steps up to $N \approx 100$ (or even to 1000 , depending on the size).

(a)

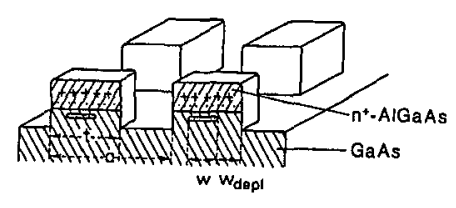

(b)



Fig. 1. Sketch of deep-mesa-etched (a) and field-effect confined (b) quantum dot arrays. In part (a) a periodic pattern is etched all the way through the AlGaAs layer into the active GaAs layer. The electrons are confined in the middle of the dots by the positively charged donors in the AIGaAs and negatively charged surface states at the side walls. Part (b) is a so-called split-gate configuration where, via a gate voltage and a varying distance between the $\mathrm{NiCr}$-gate and the channel, carriers are depleted leaving isolated quantum dots. The gate-distance modulation is achieved via a modulated photoresist layer. A low impedance $\delta$-doped layer serves as a back contact to charge the isolated dots.

Field-effect confined quantum-dot samples are sketched in Fig. 1b. On top of a heterostructure we prepare a periodic photoresist dot array by holographic lithography. The periods range from $a=500 \mathrm{~nm}$ down to $200 \mathrm{~nm}$ and the lateral photoresist dot sizes are about half the period with a height of about $100 \mathrm{~nm}$. An $8 \mathrm{~nm}$ thick semitransparent $\mathrm{NiCr}$ gate of $4 \mathrm{~mm}$ diameter is evaporated onto the photoresist structure. With a negative gate voltage we can confine the electrons under the photoresist dots and vary the number of electrons [5-7]. This is the advantage of field-effect confined dots. The disadvantage is that the confinement energy, due to the large separation of the gate with respect to the two-dimensional electron system (2DES) in typical heterostructures, is relatively small. For the same reason, also the potential is usually very smooth, parabolic as we will see below. Another disadvantage is that under illumination the gate becomes in most cases leaky, so it is not possible to do Raman spectroscopy. 


\section{FIR spectroscopy}

For our experiments we use Fourier transform spectrometers or FIR lasers which are connected to superconducting magnets. We evaluate, in gated structures, the normalized transmission of unpolarized radiation, $T\left(V_{\mathrm{g}}\right) / T\left(V_{\mathrm{t}}\right)$, where $V_{\mathrm{t}}$ is the threshold voltage at which the dots are totally depleted. In etched samples we normalize the spectra to the transmission of a reference field, where $T\left(B_{\text {ref }}\right)$ has a flat response in the interesting frequency regime. Usually, the magnetic field is oriented perpendicular to the sample. The temperature is $2 \mathrm{~K}$. The active size of the array is typically 3 to $4 \mathrm{~mm}$ in diameter, so we measure about $10^{8}$ to $10^{9}$ dots simultaneously.

Experimental FIR transmission spectra for a sample with period $a=500 \mathrm{~nm}$ are displayed in Fig. 2. At a gate voltage of $V_{\mathrm{g}}=-0.8 \mathrm{~V}$ there are about $N=50$ electrons per dot, as determined via the known transmission matrix elements from the integrated absorption strength. At $B=0$, we observe one resonance at about $30 \mathrm{~cm}^{-1}(=3.7 \mathrm{meV})$. For $B>0$ this resonance splits into two. The dispersion for these two resonances is depicted in Fig. 3a, the lower branch decreases with increasing magnetic field, the upper branch increases with increasing $B$ and approaches the cyclotron resonance frequency $\omega_{\mathrm{c}}=e B / \mathrm{m}^{*}$. In Fig. $3 \mathrm{~b}$ we also



Fig. 2. FIR transmission measured at various magnetic fields for a quantum-dot array with a period of $a=500 \mathrm{~nm}$ and a gate voltage corresponding to $N=50$ electrons per dot (from $[7,8]$ ). ES is the position of the electron system. 


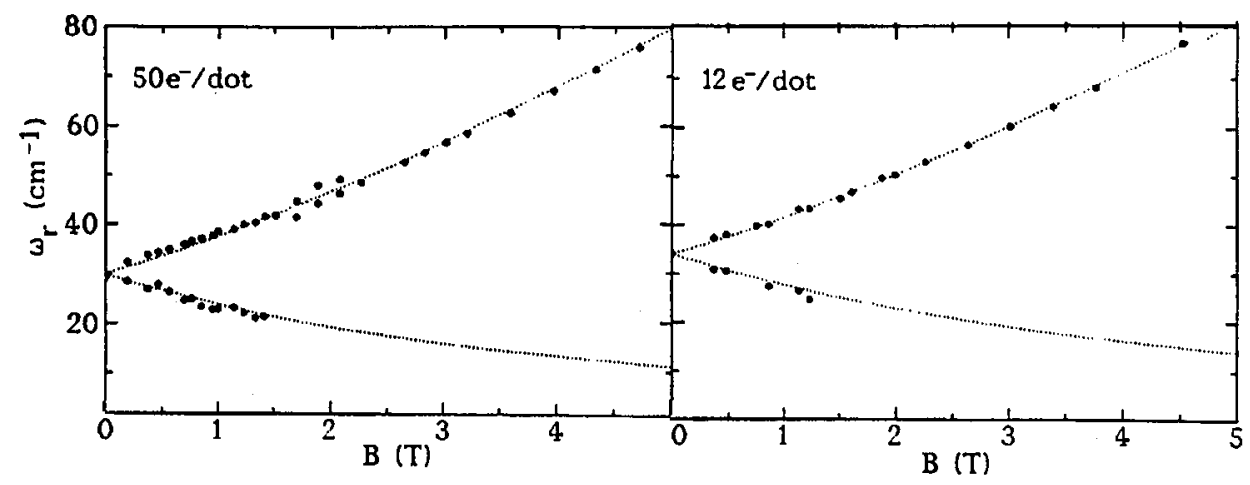

Fig. 3. Magnetic field dispersion of the resonance frequency in a quantum-dot array with a period of $a=500 \mathrm{~nm} . N=50$ (left) and $N=12$ (right) electrons per dot, respectively (from $[7,8]$ ).

plotted the dispersions for another gate voltage, $V_{\mathrm{g}}=-1.05 \mathrm{~V}$, where we have $N=12$ electrons per dot. Figure 3 shows that these dispersions do not depend significantly on the number of electrons per dot. There is only a small shift of the $B=0$ frequency to higher energies with decreasing $N$. These dispersions have been observed in many experiments, not only for quantum dots with a small number of electrons $[3,4,6,7]$, but similarly also in finite-size 2DES with much larger dimensions and electron numbers [10-12].

One can explain this dynamic response starting from two different models: (i) transitions in an "atom" including many-electron effects, and (ii) from mean-field approaches, for example, from a Hartree-Fock calculation of effective single-particle energies. These single-particle energies are not directly observed (see discussion below). The dynamical excitations are renormalized due to collective effects and represent collective charge density (plasmons, CDE), spin density (SDE) or mixed excitations. We will make use of mean field approaches later for systems with many electrons. We start with the "atomic" model and first have to realize that the external confinement potential for electrons in a field-effect and etched confined quantum dot has a nearly parabolic shape. In a simplified way we can see this from the following model. Due to the strong original 2D confinement the electron can only move in the $x-y$ directions. A positively charged layer of 2D density $N_{\mathrm{d}}$ and width $w$ of donors in the AlGaAs-GaAs layer or, in a gated structure, from a gate voltage, holds the electron in an equilibrium position. Moving the electron in the $x$ direction produces a force approximately linear in the displacement, $F=-K x$, which gives rise to a parabolic confinement potential $V(x)=\frac{1}{2} K x^{2}$. We can actually calculate the force constant $K$ and thus the eigenfrequency $\Omega_{0}^{2}=K / m^{*}$ as well as deviations from the parabolic potential by integrating the electric fields from the charged layer at the position of the electron. We first consider one electron, i.e., the one-electron Schrödinger equation in a magnetic field $B$ for a potential $V(r)=\frac{1}{2} m^{*} \Omega_{0}^{2} r^{2}$ and calculate the energy eigenvalues [13] and transition matrix elements. One finds that dipole allowed transitions have transition energies 


$$
\Delta E^{ \pm}=\sqrt{\hbar^{2} \Omega_{0}^{2}+\left(\hbar \omega_{\mathrm{c}} / 2\right)^{2}} \pm \hbar \omega_{\mathrm{c}} / 2 .
$$

This dispersion is exactly observed in our experiments as demonstrated by the fits in Fig. 3.

We do, however, not only have one electron in a quantum dot, but several. In this case electron-electron (ee) interaction should strongly influence the energy spectrum and the dynamic response. For a small number of electrons per dot it is possible to calculate many-electron wave functions and energy states in a parabolic or square well confinement [14-17]. In Fig. 4 we compare the energy spectrum of two noninteracting electrons with the interacting two-particle energy spectrum in the same parabolic confinement [16]. The many-electron energy spectrum is governed by two energies: the quantum confinement energy, $E_{\mathrm{q}}=\hbar^{2} / \mathrm{m}^{*} l_{0}^{2}$, and the Coulomb energy, $E_{\mathrm{C}}=e^{2} /\left(4 \pi \varepsilon \varepsilon_{0} l_{0}\right)$. Here $l_{0}=\sqrt{\hbar / m^{*} \Omega_{0}}$ is the confinement length of the harmonic oscillator. With increasing $l_{0}$ the Coulomb energy becomes increasingly important with respect to the confinement energy. The scale is set by the effective Bohr radius $a^{*}$ which is $10 \mathrm{~nm}$ in GaAs. The Coulomb energy $E_{\mathrm{C}}$ reflects the energy which is required to "squeeze" the second electron into the quantum dot. This energy is represented in Fig. 4 by the increase in the lowest energy level of the two-particle spectrum with respect to the noninteracting spectrum. Due to the ee-interaction some of the degeneracies of the one-particle spectrum are now lifted giving rise to a complex excitation spectrum.

The unique and important point of a parabolic confinement is, however, that the only allowed dipole transition ( $\hbar \Omega_{0}$ in Fig. 4 ) has exactly the same energy as

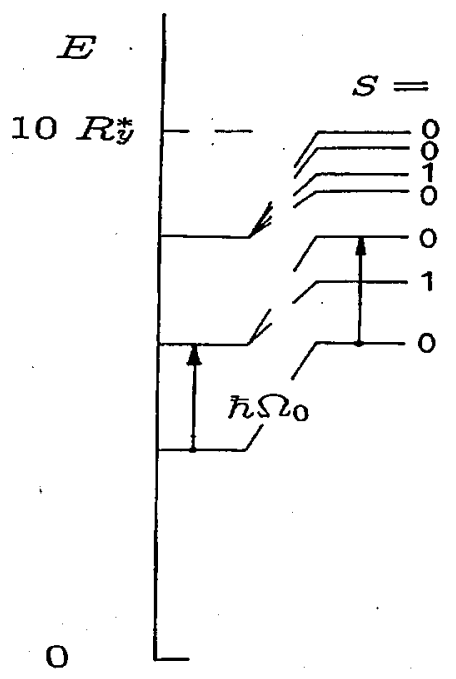

Fig. 4. The energy levels for two independent (left) and for two interacting electrons ("quantum-dot-He") in a parabolic confinement [16]. The indicated dipole-allowed transition (arrow) for quantum-dot-He has exactly the same energy as for quantum-dot- $\mathrm{H}$, i.e., $\hbar \Omega_{0} . S=0$ and $S=1$ denote singlet and triplet states, respectively. Ry* is the effective Rydberg energy. 
in the noninteracting case. Moreover, the result that the dipole-allowed transition in a parabolic confinement is exactly the resonance frequency of the one-particle spectrum, also holds for an arbitrary number of electrons, $N$. It has been shown for quantum wells [18] and quantum dots [15] with parabolic confinement, that the Hamiltonian for $N$ electrons, i.e., including ee-interaction, separates into a center-of-mass (CM) motion and into relative internal motions. The CM motion solves exactly the one-electron Hamiltonian and is the only allowed optical dipole excitation. Thus, the optical dipole response of a quantum dot with parabolic confinement represents a rigid collective $C M$ excitation at the frequency of the bare external potential, i.e., for $B=0$ at $\hbar \Omega_{0}$. This also means that for a parabolic confinement, dipole excitation frequencies are insensitive to ee-interactions and independent of the electron density in the dot. This is indicated by the small effect of the gate voltage and related number of electrons on the resonance position in Fig. 2 and has first been observed by Sikorski and Merkt [3]. The result that the dipole excitation in a parabolic confinement is not affected by ee-interactions is a generalization of Kohn's famous theorem [19], which says that the cyclotron frequency in a translational invariant system is not influenced by ee-interactions.

It is now the challenge for the experimentalist to realize quantum dot arrays with small numbers of electrons per dot to test such theoretical predictions. Indeed, we have prepared field-effect confined quantum dot arrays with well defined numbers of electrons in each individual dot [7]. The FIR transmission experiments on a field-effect confined quantum-dot array (Fig. 5) show a stepwise increase in the integrated absorption strength in its dependence on the gate voltage $V_{\mathrm{g}}$ indicating, because of sum rules, directly the incremental charging of each individual of the $10^{8}$ dots in the array with successively $N=1,2,3 \ldots$ electrons. The important

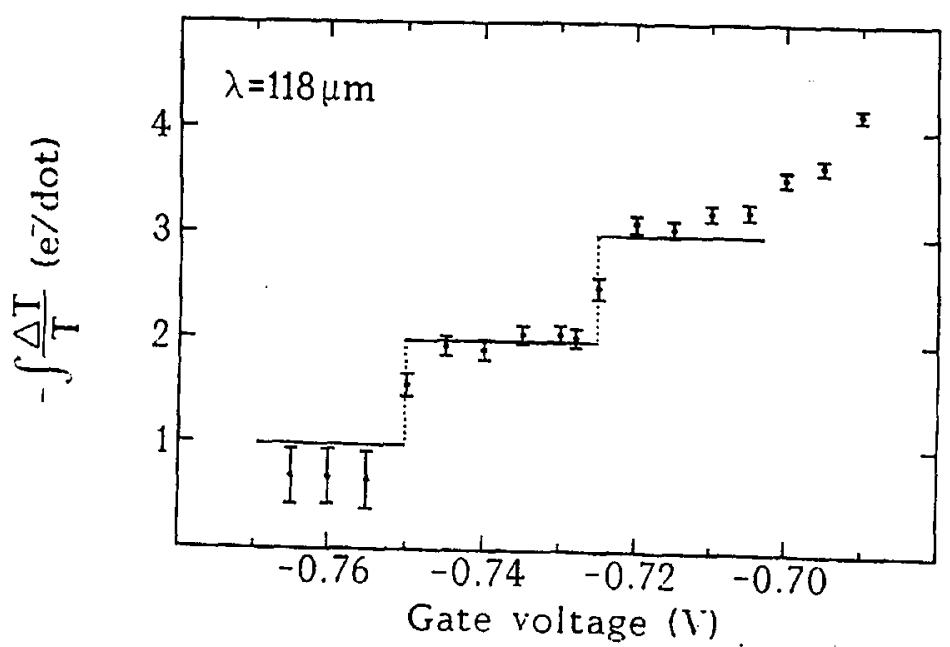

Fig. 5. Integrated absorption strength vs. gate voltage $V_{\mathbf{g}}$. The step-wise increase in the absorption strength indicates the incremental occupation of the dots with $N=1,2$, and $3 \mathrm{e}^{-} /$dot with increasing $V_{\mathbf{g}}$ (from Ref. [7]). 
aspect of this work was the preparation of very small quantum dots with a high Coulomb charging energy. The Coulomb charging energy is the energy which is required to add one additional electron into the dot. If this energy is larger than the local fluctuations of the threshold voltage, which arise from randomly distributed ionized donors and impurities in the heterostructure, it stabilizes a well defined number of electrons in a dot. This Coulomb energy can be estimated from the charging characteristic. We find that it takes about $\Delta V_{\mathrm{g}}=30 \mathrm{mV}$ increase in the gate voltage to increase the number of electrons per $\operatorname{dot}$ from $N=2$ to $N=3$. This determines a capacitance $C=e / \Delta V_{\mathrm{g}}=5 \times 10^{-18} \mathrm{~F}$ and a Coulomb energy $E_{\mathrm{C}}=e^{2} / 2 C=15 \mathrm{meV}$. The Coulomb energy for the transition from one to two electrons can also be estimated in Fig. 4 from the increase in the two-particle energy with respect to the energy of two noninteracting electrons.

\section{Overcoming Kohn's theorem}

We have learned from the generalized Kohn theorem that in parabolic quantum dots the resonance frequency is governed by the bare confinement energy $\hbar \Omega_{0}$ and is not sensitive to internal degrees of freedom and ee-interactions. However, with sophisticated technology it is possible to create quantum dots with deviations from the parabolic shape. In this case it is possible to observe various kinds of fine structures which reflect relative internal motions of the electrons. For example, energetically higher modes and resonant anticrossing of these modes have been observed [4]. This type of anticrossing occurs in dots which do not have a circular symmetry. Another type of fine structure is observed in Fig. 3. In the dispersion for the 50-electron dots we find that the upper branch exhibits a resonant anticrossing at $B=2 \mathrm{~T}$ and $\omega_{\mathrm{r}}=45 \mathrm{~cm}^{-1}$. Inspection of more spectra shows that this type of anticrossing is close to the energies $2 \hbar \omega_{c}$ or $3 \hbar \omega_{c}$. This interaction is not allowed in a strictly parabolic confinement potential and indicates deviations from this shape. It resembles a similar interaction of 2D plasmons with the harmonics of the cyclotron resonance, the so-called "Bernstein" modes [20]. Similar interactions have also been observed in other types of microstructures, i.e., antidots [21] for the mixed-mode 1D plasmons in wires [22], as well as for the CM mode of quantum wires [23]. One can explain this interaction theoretically in random phase approximation (RPA) calculations on dots with nonparabolic confinement (also for circular symmetry) [24]. The unique feature of the interaction here is that, depending on the strength of the nonparabolicity, this interaction does not occur directly at $2 \omega_{\mathrm{c}}$ but can also be shifted to significantly smaller energies, somewhere in the regime between $\omega_{\mathrm{c}}$ and $2 \omega_{\mathrm{c}}$ ! This is in contrast to the observation of the Bernstein modes on 2DES and seems to be a special behavior of lower-dimensional systems. Experiments and theory of the Bernstein modes in quantum dots and wires are discussed extensively in [25]. Another type of fine structure can be generated in magnetic fields tilted with respect to the sample normal. In this case coupling and anticrossing with the intersubband resonance arising from the original confinement in the $z$ direction occurs [8].

We will not elaborate on these experiments here, but we like to discuss another very interesting ee-interaction phenomenon, the formation of compressible 
and incompressible states in quantum dots and wires and its investigation with FIR spectroscopy. The formation of compressible and incompressible states is one of the currently most intensively discussed phenomena in the field of mesoscopic systems and semiconductor microstructures. It was proposed by Beenakker [26] and Chklovskii et al. [27] that the electron density near the edge of a 2DES in the presence of a magnetic field $B$ exhibits, to a certain degree, a step-like density profile $N_{\mathrm{s}}(r)$ arising from the self-consistent and $B$-dependent formation of incompressible stripes, where the $2 \mathrm{D}$ density $N_{s}(r)$ is fixed at integer filling factors $\nu$, i.e., $N_{\mathrm{s}}(r)=\nu e B / h$, and compressible stripes, where $N_{\mathrm{s}}(r)$ can vary. $r$ is the direction perpendicular to the edge in the $2 \mathrm{D}$ plane. This many-body effect has an important influence on the transport properties in the integer and fractional quantum Hall effect.

We have prepared dot and antidot arrays starting from modulation-doped $\mathrm{AlGaAs}-\mathrm{GaAs}$ double-layered quantum-well structures [9]. An antidot array is a reverse structure with respect to a dot array and can be prepared by etching an array of geometrical holes into an original 2DES [21]. Three doping layers, one on the top of the upper quantum well, one in the barrier, and one below the lower quantum well were separated by spacers on each side. The dot array had a period of $a=800 \mathrm{~nm}$. The geometrical radius of the dots was $400 \mathrm{~nm}$ which contain about 750 electrons per well in each dot. The double-layer dots and antidots with high electron densities in our experiments increase the signal strength and, moreover, the three doping layers increase the external potential and thus the frequencies, in particular also of the low frequency mode. They also produce a nonparabolic hard-wall-type confining potential which makes our experiments sensitive to ee-interactions.

The experimental dispersion for the dot array is depicted in Fig. 6. We observe again two strong modes. What is surprising and was observed for the first time in [9] for dot and antidot arrays, is the oscillatory behavior of the resonance frequency. If we plot the frequencies of the low-frequency modes versus the inverse magnetic field $B^{-1}$, we find that both the maxima and minima of the resonance frequency are periodic in $B^{-1}$. We can relate them to the filling factor $\nu=N_{\mathrm{s}} h / e B$. We find that for dots, minima in the resonance frequency occur at half filled Landau levels ( $\nu$ odd) and maxima for fully occupied Landau levels ( $\nu$ even). Spin splitting is not resolved for our experimental conditions. The interesting finding is that for the antidots the maxima and minima of the eigenfrequencies are again filling-factor related, however, with an opposite behavior as compared to the dot array.

To explain this behavior we have performed self-consistent Hartree calculations of the eigenstates and the density profile for a single-layer dot containing $N=60$ electrons. We assume a degeneracy of $e B / h$ per Landau level and model the nonparabolic potential by $V(r)=\frac{1}{2} \hbar \omega_{0}\left[\left(r / l_{0}\right)^{2}+a\left(r / l_{0}\right)^{4}\right]$, with $\hbar \omega_{0}=3.37 \mathrm{meV}$, $m^{*}=0.067 m_{\mathrm{e}}$ and $a=0.0674 ; T$ is $1.0 \mathrm{~K}$. The calculation techniques are described in [25]. In Fig. 7a we show the resulting density profile $N_{s}(r)$. At $B=4.9 \mathrm{~T}$ we have a pronounced formation of regions with flat densities indicating the incompressible regimes. For $B=3.7 \mathrm{~T}$, when the filling factor in the middle of the dot does not have an even value, the density profile smooths out, indicating a suppression of the incompressible states. Starting from the Hartree energies we 


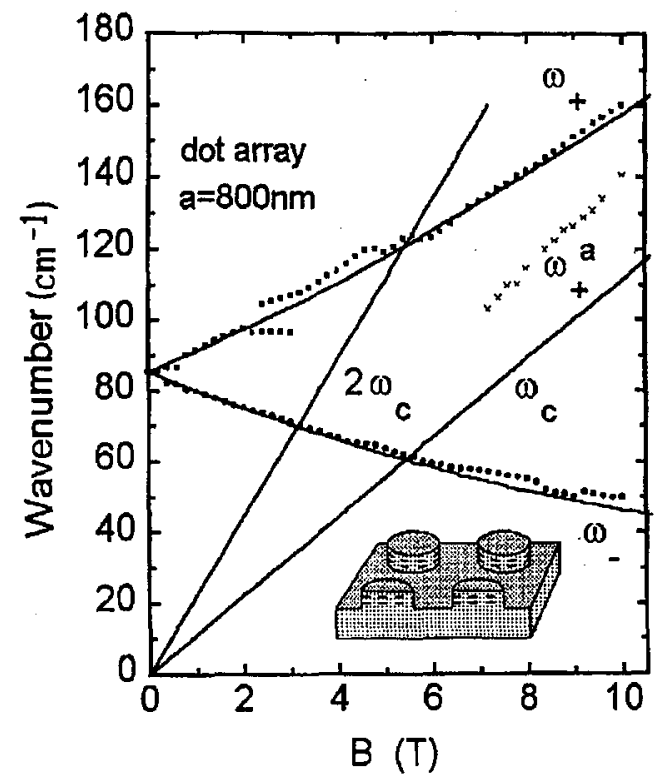

Fig. 6. Experimental dispersions of the resonance frequencies in a dot array. The low-frequency mode $\left(\omega_{-}\right)$exhibits well pronounced oscillations [9]. The straight curves represent a calculated dispersion $\omega_{ \pm}^{\text {ca }}$ using Eq. (1) with $\omega_{0}=86 \mathrm{~cm}^{-1}$. The mode labeled $\omega_{+}^{\mathrm{a}}$ is the acoustic mode of the double-layered dot array.


Fig. 7. (a) Hartree calculation of the density profile in a quantum dot in units of $10^{11} \mathrm{~cm}^{-2}$. The flat regions for $B=4.9 \mathrm{~T}$ indicate the incompressible regimes. (b) Dispersion within the Hartree/RPA for the nonparabolic potential compared to the classic result for the parabolic potential. (c) Ratio of the RPA and the result for a parabolic potential $\omega_{-} / \omega_{-}^{\text {pa }}$, vs. $B^{-1}$ (from [9]). 
calculated within RPA the $\omega_{-}$FIR resonance frequency. In Fig. 7b we see that the filling-factor-dependent formation of the compressible and incompressible stripes leads indeed to frequency oscillations with exactly the same phase as in the experiment, e.g., a maximum in the resonance frequency at full filling in the "bulk", if we call the center of the dot the "bulk" region. This confirms our experiments and interpretation. An oscillatory behavior has also been calculated by Darnhofer et al. [28].

Besides using a nonparabolic potential, we can break Kohn's theorem, and thus study the internal ee-interaction also by various others means. Darnhofer and Rössler [29] have shown that a nonparabolic band structure, meaning a nonquadratic $k$-dependence in the energy dispersion in Eq. (1), produces a quite similar type of anticrossing behaviors as discussed above.

We would like to note that we restricted ourselves here to quantum dots that where prepared starting from conventional heterostructures. There are also other systems, e.g., very interesting effects like shell structures have recently been observed in quantum dots prepared by novel self-assembling techniques [30].

\section{Raman spectroscopy}

Another powerful tool to investigate the electronic excitations in modulation-doped quantum dots is resonant Raman spectroscopy. In zinc-blende-type semiconductors one can excite CDE's (plasmons), whose energies are renormalized with respect to single-particle energy spacings due to direct and exchange Coulomb interactions. One can also excite SDE's [31], which are only affected by exchange interaction and are therefore in most cases redshifted with respect to the corresponding single-particle energies [32,33]. The CDE's and SDE's can be distinguished by polarization selection rules. CDE's are observed if the polarizations of the incoming and scattered light are parallel to each other (polarized geometry) and SDE's can be observed if the polarizations are perpendicular (depolarized geometry). A very interesting point is that in the Raman spectroscopy one can easily vary also the wave vector $q$ of the excitation which gives valuable additional information. In particular, for $q \neq 0$ we have a non-dipole excitation which means that Kohn's theorem is broken. There is a number of interesting Raman investigations of modulation-doped quantum dot structures with [34] and without [33, 35] external magnetic field.

Here we would like to present briefly some recent measurements by Schüller et al. [36] of AlGaAs-GaAs quantum dots, where we have probed in one and the same quantum dot sample the spectrum of collective CDE's and SDE's in dependence on the transferred lateral wave vector $q$ and magnetic field $B$, to elucidate the potential of the Raman technique. An extended recent review on the Raman spectroscopy of quantum dots and wires was given in [37].

Figure 8 shows the experimental dispersions in an external magnetic field. The solid symbols correspond to SDE's and the open symbols to CDE's. We find that in a magnetic field the polarization selection rules are weakened in a complicated manner. Therefore plasmons can also be observed in the depolarized geometry. The open triangles in Fig. 8 correspond to CDE's which are most pronounced 


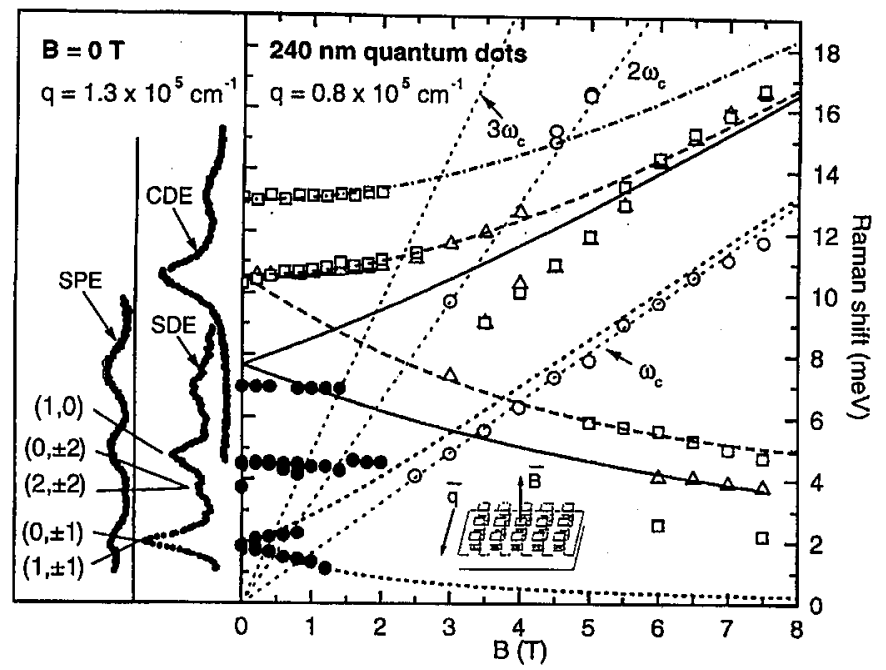

Fig. 8. Magnetic field dispersions of the observed modes in a quantum dot sample measured with the Raman spectroscopy. Solid symbols mark SDE and open symbols correspond to magnetoplasmon modes. In the left panel spectra of SDE and CDE for $B=0$ are displayed. The numbers in the brackets, $(\Delta n, \Delta m)$, give the changes in radial $(\Delta n)$ and azimuthal $(\Delta m)$ quantum numbers for the observed transitions (from [36]).

in depolarized spectra and the open squares to CDE's which are dominant in polarized scattering geometry. For illustration, the spectra of the SDE's and CDE's at $B=0 \mathrm{~T}$ and large $q$ are displayed in the left panel of Fig. 8. The high-frequency modes which are plotted with open symbols are the same excitations that we also see in FIR experiments, however with complementary excitation strengths. The set of modes with the lowest frequencies, which starts at about $8 \mathrm{meV}$ for $B=0$, is the strongest mode in FIR, but very weak in the Raman experiments. Higher frequency mode pairs, starting at $10 \mathrm{meV}$ and $15 \mathrm{meV}$ for $B=0$, are strong in the Raman but only very weak in FIR spectroscopy and are only observed for nonparabolic confinement, as required by Kohn's theorem. For Raman, the finite $q$ values break the theorem for the lowest mode pair. It is intrinsically broken for the high-frequency plasmon branches.

An interesting finding in Fig. 8 are the SDE's which cannot be observed in FIR experiments. They occur at lower frequencies and are much closer to the single-particle energies of the screened Hartree-Fock potential. These excitations have been intensively discussed in the original paper [36]; in particular, they allow a detailed study of a quasi-atomic fine structure in these artificial atoms. Another important finding is that, under strongly resonant conditions, one observes in the Raman spectroscopy also the so-called single-particle excitations (SPE's), which occur very close to the single-particle energies of the Hartree-Fock potential [32, 33]. Conceptionally, if we use the many-électron picture as demonstrated for two electrons in Fig. 4, there are no single-particle energies in this model. Indeed, it has been shown for quantum wires [38] and quantum dots [39] that 
under strongly resonant conditions modes are excited, which are very close to single-particle resonances, nevertheless exhibiting small shifts due to collective interactions.

We thank many colleagues, as listed in the original references, which have been working with us on the different projects and acknowledge financial support by the German Science Foundation through the Graduiertenkolleg "Nanostrukturierte Festkörper" and Sonderforschungsbereich 508 "Quantenmaterialen", and the Icelandic Science Foundation.

\section{References}

[1] D. Heitmann, J.K. Kotthaus, Phys. Today 46/6, 56 (1993).

[2] D. Heitmann, in: Confined Electrons and Phonons, Series B: Physics, Eds.

E. Burstein, C. Weisbuch, Vol. 340, Plenum Press, New York 1995, p. 305.

[3] Ch. Sikorski, U. Merkt, Phys. Rev. Lett. 62, 2164 (1989).

[4] T. Demel, D. Heitmann, P. Grambow, K. Ploog, Phys. Rev. Lett. 64, 788 (1990).

[5] J. Alsmeier, E. Batke, J.P. Kotthaus, Phys. Rev. B 41, 1699 (1990).

[6] A. Lorke, J.P. Kotthaus, K. Ploog, Phys. Rev. Lett. 64, 2559 (1990).

[7] B. Meurer, D. Heitmann, K. Ploog, Phys. Rev. Lett. 68, 1371 (1992).

[8] B. Meurer, D. Heitmann, K. Ploog, Phys. Rev. Lett. 48, 11488 (1993).

[9] K. Bollweg, T. Kurth, D. Heitmann, V. Gudmundsson, E. Vasiliadou, P. Grambow, K. Eberl, Phys. Rev. Lett. 76, 2774 (1996).

[10] S.J. Allen, Jr., H.L. Störmer, J.C. Hwang, Phys. Rev. B 28, 4875 (1983).

[11] D.C. Glattli, E.Y. Andrei, G. Deville, J. Poitrenaud, F.I.B. Williams, Phys. Rev. Lett. 54, 1710 (1985).

[12] D.B. Mast, A.J. Dahm, A.L. Fetter, Phys. Rev. Lett. 54, 1706 (1985).

[13] V. Fock, Z. Phys. 47, 446 (1928).

[14] G.W. Bryant, Phys. Rev. Lett. 59, 1140 (1987).

[15] P. Maksym, T. Chakraborty, Phys. Rev. Lett. 65, 108 (1990).

[16] U. Merkt, J. Huser, M. Wagner, Phys. Rev. B 43, 7320 (1991).

[17] D. Pfannkuche, R.R. Gerhardts, Phys. Rev. B 44, 13132 (1991).

[18] L. Brey, N. Johnson, P. Halperin, Phys. Rev. B 40, 10647 (1989).

[19] W. Kohn, Phys. Rev. 123, 1242 (1961).

[20] E. Batke, D. Heitmann, J.K. Kotthaus, K. Ploog, Phys. Rev. Lett. 54, 2367 (1985).

[21] K. Kern, D. Heitmann, P. Grambow, Y.H. Zhang, K. Ploog, Phys. Rev. Lett. 66, 1618 (1991).

[22] T. Demel, D. Heitmann, P. Grambow, K. Ploog, Phys. Rev. Lett. 66, 2657 (1991).

[23] H. Drexler, W. Hansen, J.P. Kotthaus, M. Holland, S.P. Beaumont, Phys. Rev. Lett. 54, 2367 (1985).

[24] V. Gudmundsson, R.R. Gerhardts, Phys. Rev. B 43, 12098 (1991).

[25] V. Gudmundsson, A. Brataas, P. Grambow, B. Meurer, T. Kurth, D. Heitmann, Phys. Rev. B 51, 17744 (1995).

[26] C.W. Beenakker, Phys. Rev. Lett. 64, 216 (1990). 
[27] D.B. Chklovskii, B.I. Shklovskii, L.I. Glazman, Phys. Rev. B 46, 4026 (1992).

[28] T. Darnhofer, M. Suhrke, U. Rössler, Europhys. Lett. 35, 591 (1996).

[29] T. Darnhofer, U. Rössler, Phys. Rev. B 47, 16020 (1993).

[30] M. Fricke, A. Lorke, J.P. Kotthaus, G. Medeiros-Ribeiro, P.M. Petroff, Europhys. Lett. 36, 197 (1996).

[31] For an overview, see A. Pinczuk, G. Abstreiter, in: Light Scattering in Solids V, Topics in Applied Physics, Vol. 66, Eds. M. Cardona, G. Güntherodt, Springer, Berlin 1988, p. 153.

[32] A. Pinczuk, S. Schmitt-Rink, G. Danan, J.P. Valladares, L.N. Pfeiffer, K.W. West, Phys. Rev. Lett. 63, 1633 (1989).

[33] C. Schüller, G. Biese, K. Keller, C. Steinebach, D. Heitmann, P. Grambow, K. Eberl, Phys. Rev. B 54, R17304 (1996).

[34] D.J. Lockwood, P. Hawrylak, P.D. Wang, C.M. Sotomayor Torres, A. Pinczuk, B.S. Dennis, Phys. Rev. Lett. 77, 354 (1996).

[35] R. Strenz, U. Bockelmann, F. Hirler, G. Abstreiter, G. Böhm, G. Weimann, Phys. Rev. Lett. 73, 3022 (1994).

[36] C. Schüller, K. Keller, G. Biese, E. Ulrichs, L. Rolf, C. Steinebach, D. Heitmann, K. Eberl, Phys. Rev. Lett. 80, 2673 (1998).

[37] C: Schüller, Advances in Solid State Physics, Ed. B. Kramer, Vol. 38, Vieweg Verlag, Braunschweig 1998, p. 167.

[38] M. Sassetti, B. Kramer, Phys. Rev. Lett. 80, 1485 (1998).

[39] C. Steinebach, C. Schüller, D. Heitmann, Phys. Rev. B 59, 10240 (1999). 